\title{
Low incidence of COVID-19 in the West African sub-region: mitigating healthcare delivery system or a matter of time?
}

\author{
Taofik Olatunji Bankole ${ }^{1}$ (D) Olajide Bamidele Omoyeni ${ }^{2} \cdot$ Abiodun Oluwaseun Oyebode $^{3}$. \\ David Olumide Akintunde ${ }^{4}$
}

Received: 2 May 2020 / Accepted: 5 October 2020 / Published online: 17 October 2020

(C) Springer-Verlag GmbH Germany, part of Springer Nature 2020

\begin{abstract}
Background This study examines the growth trends in the COVID-19 pandemic and fatalities arising from its complications among tested patients in West Africa. Countries around the world have employed several measures in order to control the spread of the disease. In spite of the poor state of the healthcare delivery system in West Africa, the spread of the pandemic is relatively low compared to reported cases in other regions of the world. The study addresses this phenomenon by asking the question: is the low incidence of COVID-19 in the West African sub-region a mitigating healthcare delivery system or just a matter of time? Methods The study adopted a cross-sectional time series method. Data for Ghana, Nigeria, Burkina Faso, Ivory Coast, Senegal, Niger Republic, and global data were extracted from the World Health Organization COVID-19 databank. Data were extracted in intervals of 7 days from March 15 through April 19, 2020. Data regarding the incidence growth rate and fatalities arising from COVID-19 complications were generated from the total reported cases and fatalities over specified periods. Descriptive and inferential statistical analyses were carried out using Stata version 14.

Results Results showed that the trends in growth patterns of COVID-19 for Senegal, Nigeria, Ghana, Republic of Niger, and West Africa generally followed the same fluctuating curves. The COVID-19 pandemic accounted for 92.3\%, 97.8\%, 90.3\%, $65 \%, 90.4 \%, 93.6 \%$, and $97 \%$ of complications that led to deaths of patients in Burkina Faso, Nigeria, Senegal, Ghana, Niger Republic, Ivory Coast, and West Africa, respectively. Also, the results established that there was a significant positive association between increased incidence of COVID-19 and percentage increase in fatalities arising from its complications in West Africa $(ß=0.032 ; t=12.70 ; p<0.001)$.

Conclusion The threat presently posed by COVID-19 seems to be minimal in West Africa despite the poor state of the healthcare delivery system in the region. It is unlikely, however, that the region is well prepared for the pandemic in the event that it escalates out of control with time.
\end{abstract}

Keywords Mitigation $\cdot$ COVID-19 $\cdot$ Pandemic $\cdot$ West Africa $\cdot$ Healthcare delivery $\cdot$ Fatalities

\section{Introduction}

The recent COVID-19 outbreak is the most devastating pandemic that mankind has experienced in the past ten decades.

Taofik Olatunji Bankole

oluwabamikoleolatunji@gmail.com

Olajide Bamidele Omoyeni

omoyeniolajide1@gmail.com

Abiodun Oluwaseun Oyebode

abiodun_oyebode@yahoo.com

David Olumide Akintunde

davidolumideakintunde@gmail.com
In fact, no other pandemic has claimed more human lives in the same amount of time. The severity and contagion is so high that no continent has been spared its threat. In fact, empirical reports show that over 3.06 million persons have been

1 Department of Demography and Social Statistics, Faculty of Social Sciences, Obafemi Awolowo University, Ile-Ife, Nigeria

2 Department of Chemistry, Faculty of Sciences, Obafemi Awolowo University, Ile-Ife, Nigeria

3 Department of Social Sciences and Humanities, Federal Polytechnic, Offa, Nigeria

4 Institute of Early Childhood Education, Faculty of Education, Obafemi Awolowo University, Ile-Ife, Nigeria 
infected with the virus, while about 210,000 deaths have been reported across regions of the world, including about 57,000, 27,000, 24,000 and 23,000 deaths reported as of April 28, 2020 , in the United States, Italy, Spain, and France, respectively (WHO 2020c).

Across countries, several measures have been adopted to contain the spread of the pandemic. As noted in the World Health Organization Interim Guidance for infection prevention and control, it is imperative that member countries strictly adhere to standard precautions for all infected persons, while the establishment of an infection prevention and control focal centre was also recommended, supported by nationwide and facility senior management across countries (WHO 2020a, b). In fact, these recommended strategies and practices are considered vital for nations to attain the maximum level of effectiveness in response to the COVID-19 plague. Andrea and Giuseppe (2020) posited that the COVID-19 pandemic is manageable in the foreseeable future, but this is only achievable where and if all-inclusive and stringent control measures are taken.

The spread of COVID-19 across countries in the subSaharan African region seems to have been relatively low compared to the reported cases originating from other regions of the world. In fact, as of April 18, 2020, with the exception of South Africa, no country in the sub-region has yet reached 2000 reported COVID-19 cases since the outbreak in December 2019. Specifically, only Cameroun has reported up to 1000 COVID-19 cases, closely followed by 42 cases reported in the Ivory Coast, 641 in Ghana, 627 in Niger, 557 in Burkina Faso, and 493 in Nigeria (Worldometer 2020). In all, the total COVID-19 cases across countries in Africa was estimated at 20,706, with countries in sub-Saharan Africa accounting for about $52.9 \%$ (910, 947 reported cases) of infected persons despite housing 49 out of the 56 countries (with the exclusion of Egypt, Algeria, Tunisia, Djibouti, Libya, Morocco, Sudan, and Somalia) on the African continent (Worldometer 2020).

Arguably, the spread of infection in the sub-region can be questioned. In fact, there are unclassified arguments among peoples of the sub-region that either the cases are overreported or the pandemic scarcely exists in the subcontinent. In contrast, it is also argued that COVID-19 cases are being reported in many of the countries in the sub-region. Specifically, Melinda Gates, in her wake-up call to African leaders, lamented that deaths from COVID-19 in the region may escalate out of control unless drastic and immediate measures are taken (Sahara Reporters 2020). Gates maintained that the logical reason for the present lower number of reported cases $(13,814)$ and deaths (747) is the lack of testing kits. She argued that it was often impossible to practice physical distancing in many African towns and slums (Sahara Reporters 2020). Indeed, personal hygiene and physical distancing may be difficult to adhere to not only because of the widespread poverty in many of the sub-Saharan African countries, but also due to the communal way of life in traditional African settings.

Indeed, the possibility of underreported cases of COVID19 is not likely restricted to countries of sub-Saharan Africa; the outcomes of data-driven modeling analysis in China showed a high likelihood that cases of infection were unreported (Zhao et al. 2020). Evidence from simulated reported cases revealed a significantly larger number of observed versus actual reported cases within a span of 16 days (Zhao et al. 2020). Closely related to this, Wu et al. (2020) noted that, in defiance of early expectations, the once-assumed epidemic is not only growing steadily and exponentially in many Chinese communities, but has grown into a pandemic across the continents of the world. A retrospective study of the predicted outcomes of COVID-19 infection from a simulated process revealed a significant likelihood of underreported cases of the virus (Chen et al. 2020).

In agreement with the aforementioned, there is a clear indication that cases of COVID-19 in the sub-Saharan region are underreported, while many cases are surely not reported at all. The high possibility of unreported cases could be argued from the perspective of the ratio of tests carried out to reported cases of infections. The rate of tests per population is generally low across countries of the region-while the ratio was as low as 194 per 1 million population in the Republic of Niger, the ratios were estimated at 60, 35, 29, 22, and 7 persons per 1 million population in Ethiopia, Nigeria, Mozambique, Malawi, and Burundi, respectively (Worldometer 2020).

Evidence from an empirical survey revealed that personal protective equipment, administrative control, and basic healthcare facilities are not only in short supply, but are also of poor quality in the region (WHO 2020b). As noted by $\mathrm{Li}$ et al. (2020) and Zhou et al. (2020), there is a need for stringent and timely epidemiological approaches in order to restrict the rate at which the virus is spread. Could it be that these measures are being taken as part of daily routine by countries of this region? In fact, virtually all tests in healthcare facilities in the region were carried out on patients that met the suspected case definition as spelled out by the World Health Organization recommendations for COVID-19 testing (WHO 2020a).

According to Oleribe et al. (2019) and Doctor et al. (2018), practically all the sub-Saharan African countries are faced with a shortage of quality human resources, lack of healthcare financing, poor governance, and management lapses. In fact, the healthcare insurance schemes are either not available or are non-functional in many countries of the region, thereby forcing healthcare workers to risk their lives with no assurance of better lives for their loved ones in the event that they die while working to save the lives of others (Kiri and Ojule 2020; Lawumi 2020; Gautier and Ridde 2017; Olugbenga 2017). 
In spite of the weak state of healthcare facilities, shortage of well-trained medical professionals, and low level of adherence to physical distancing, the spread of COVID-19, as well as deaths arising from the viral infection, is still relatively low compared to other regions of the world with better healthcare service delivery systems and fewer slums or shanty communities. It is against this backdrop that the current study asks the question: is the low incidence of COVID-19 in the West African sub-region the mitigating effects of the healthcare delivery system or just a matter of time? The study is restricted to the sub-region of West Africa for three reasons. First, the population density of 65 persons per square kilometre makes it the most congested region of Africa (PRB 2019). Also, the life expectancy of 57 years is the lowest across regions in Africa (PRB 2019; WHO 2019). Lastly, the present state of healthcare service delivery is not only poor but is inadequate for the population of the region (Kiri and Ojule 2020; WHO 2019).

The six countries with the highest reported cases of COVID-19 infections as on April 15, 2020, were purposefully and specifically selected for the study, although the aggregated trends for the sub-region (West Africa) and the world at large were also investigated. Hence, the specifically selected countries for the study include Burkina Faso, Ivory Coast, Niger, Ghana, Nigeria, and Senegal.

\section{Theoretical Approach: Socio-ecological Theory}

According to McLeroy et al. (1988), socio-ecological theory is a comprehensive approach to behavioural action, and such detailed action is typically influenced by factors on multiple levels. The proponents of the theory maintain that its adaptation must be built on the agreement between the benefactor and the beneficiaries of the proposed action. Therefore, the fundamental aspects of the ecological approach, with the goal of reducing challenges of subjugation, and particularly repression, is the active involvement of the target population in problem classification, selection of targets for change and appropriate intervention, implementation, and appraisal. The sociological model according to McLeroy et al. (1988) is segmented into five classes, including intrapersonal factors, interpersonal processes, institutional or organizational factors, community factors, and public policy.

Our study is supported by the socio-ecological theory. The theory is found relevant since it explains the effect of environmental dynamics on human behaviour, with the primary aim of identifying environmental interference to addressing the phenomenon or subject matter. In the context of preventing and minimizing the spread of COVID-19 in the sub-region of West Africa, it is recommended that governments, stakeholders, and the entire population at large adopt measures that will enable then to become immersed in the immediate environment - while personal hygiene such as regular washing of hands and wearing of masks covering the nose and mouth are encouraged, social and physical distancing is also encouraged - with the governments expected to provide extra support for improving, expanding, and making healthcare facilities accessible for testing (WHO 2020d).

Also, the willingness and the extent to which the recommended measures were adhered to across the West African sub-region is strongly dependent on the ability of the governments to provide the needed COVID-19 test kits across strategically well-equipped healthcare facilities, as well as the provision of palliatives in the form of food, energy, drugs, and other basic necessities, especially to those in dire need of them. Also, the existence of functional or establishment of viable social and health insurance schemes is needed, as this will encourage healthcare workers and others who are directly involved in containing the spread of the virus to willingly perform their assigned duties.

Deductively, the extent to which the governments of nations in the West African sub-region are able to provide palliatives that will compel their nationals to totally comply with the pronounced lockdown in some communities, provide the population with a regular and accessible supply of face masks, equip healthcare facilities with COVID-19 test kits and ventilators, and provide or implement social and healthcare insurance, will determine how well the region is prepared to contain the spread of the virus in case the unexpected arises. Hence, there is no doubt that the socio-ecological model provides insight into a theoretical approach to tie the rate at which the infection is spread to the state of healthcare service delivery and socio-ecological factors preventing the population from totally complying with the recommended interim guideline issued by the World Health Organization.

\section{Methods}

The study adopted a cross-sectional time series method. Secondary data were sourced from the World Health Organization COVID-19 databank. Data including daily reports on the total reported cases of COVID-19 infection and the number of related patient deaths were extracted for Burkina Faso, Ivory Coast, Niger, Ghana, Nigeria, and Senegal over the time span covered in this study. The extracted data were entered into an Excel spreadsheet and later exported to Stata analysis software. The incidence growth rate for the total number of reported cases was generated from two consecutive intervals (weekly), with the former retrieved data adopted as the base value (denominator) for the specified total reported cases of infection, while the latter was used as the numerator value. Also, the death rate was generated by adopting the former retrieved total death data for each of the 
specified time intervals (weekly) for specified dates as the base value (denominator), and the latter retrieved data was adopted as the numerator value. Descriptive (figures) and inferential (linear regression, correlation) analytic techniques were adopted to analyse the trend variations, cause-effect association, and strength of the relationship. Stata version 14 software was employed.

\section{Results}

Figures $1,2,3,4,5,6,7$, and 8 show the trends in the percentage increase in the rate of reported cases of COVID-19 in Burkina Faso, Ivory Coast, Senegal, Nigeria, Ghana, Republic of Niger, West Africa, and the world, respectively. The results show that the growth patterns for Burkina Faso and globally followed the same pattern of growth. Although there are increases in reported cases, a decline in growth across intervals is found, as presented in Figs. 1 and 8, respectively. In contrast, the prevalence rates for Ivory Coast, Senegal, Nigeria, Ghana, Niger Republic, and West Africa as presented in Figs. 2, 3, 4, 5, 6 and 7 generally follow the same patterns; fluctuations in growth patterns were recorded in these four countries, as well as in the West African sub-region.

For instance, reported cases of COVID-19 for Burkina Faso as captured in Fig. 1 showed a drop in the infection rate from the initial recorded growth of $2300 \%$ in $(\mathrm{Tn}+1)$ to $202.6 \%$ in $(\mathrm{Tn}+2)$ and a further decline to a growth rate of $106.9 \%$ in $(\mathrm{Tn}+3)$. The infection rate subsequently dropped to $46 \%$ in $(\mathrm{Tn}+4)$, and further declined to $30 \%$ by the end of $(\mathrm{Tn}+5)$. For the Ivory Coast, as reported in Fig. 2, the incidence growth rate of COVID-19 indicated that the reported cases initially increased from $200 \%$ in $(\mathrm{Tn}+1)$ to $1445.6 \%$ in $(\mathrm{Tn}+2)$, and subsequently dropped to $75 \%$ in $(\mathrm{Tn}+4)$. Also, the results indicated that the growth rate for the reported

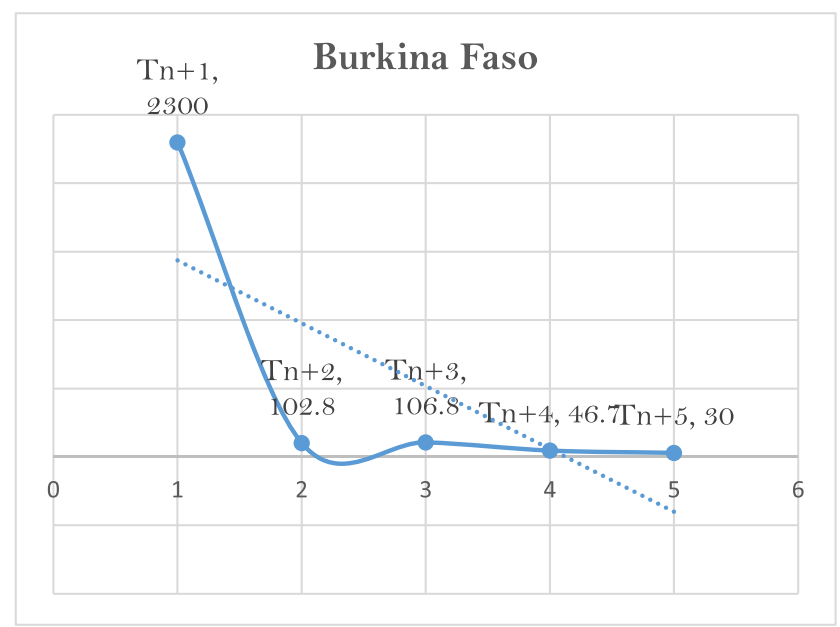

Fig. 1 Trends in COVID-19 cases in Burkina Faso

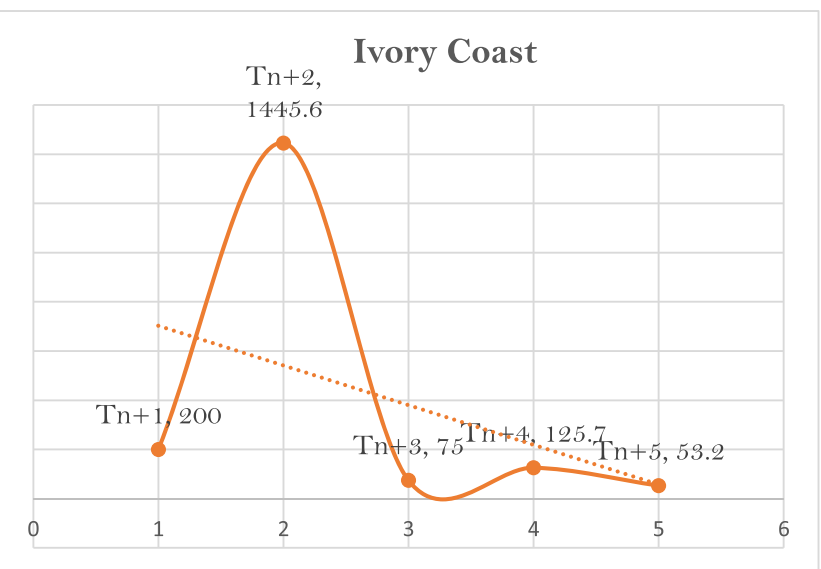

Fig. 2 Trends in COVID-19 cases in Ivory Coast

incidence of COVID-19 in the country increased to $125.7 \%$ in $(\mathrm{Tn}+4)$ and then dropped to $53.2 \%$ in $(\mathrm{Tn}+5)$.

The results as captured in Fig. 3 indicate that the prevalence of reported COVID-19 for Senegal dropped from an initial growth rate of $166.7 \%$ recorded in $(\mathrm{T} n+1)$ to $112.5 \%$ in $(\mathrm{Tn}+2)$. A further drop to $84 \%$ was recorded in $(\mathrm{Tn}+3)$ and a subsequent decline to $26 \%$ in $(\mathrm{Tn}+4)$. However, the consistent declining growth rate pattern for the country over time was reversed in $(\mathrm{Tn}+5)$, which showed an increase of $32 \%$. The results for Nigeria as captured in Fig. 4 indicate that the country's reported cases of COVID-19 grew by $1000 \%$ in $(\mathrm{Tn}+1)$. Although the growth rate dropped to $195.5 \%$ in $(\mathrm{Tn}+2)$, an upward trend of $223.1 \%$ was reported in $(\mathrm{Tn}+3)$, followed by a decline to $54.1 \%$ in $(\mathrm{Tn}+3)$. This decline in $(\mathrm{Tn}+4)$ was followed by an upward trend to $97.2 \%$ by the end of $(\mathrm{Tn}+5)$.

The growth rate for reported cases of COVID-19 for Ghana is nearly the same as that for Nigeria. The results as captured in Fig. 5 indicate a growth rate of $1850 \%$ in $(\mathrm{Tn}+1)$, which subsequently dropped to $621.1 \%$ in $(\mathrm{Tn}+2)$. The results indicate a sporadic decline - this was estimated at $49.6 \%$ in ( $\mathrm{Tn}+$

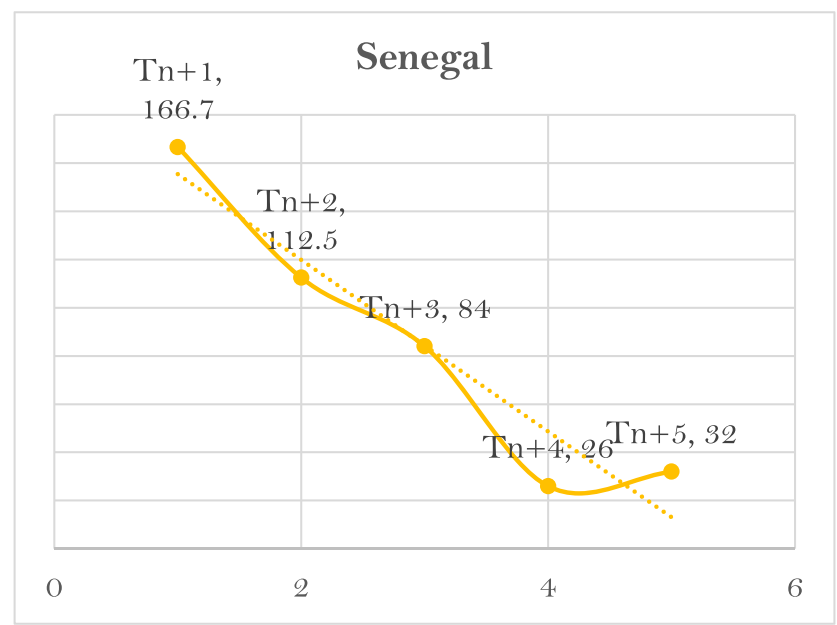

Fig. 3 Trends in COVID-19 cases in Senegal 


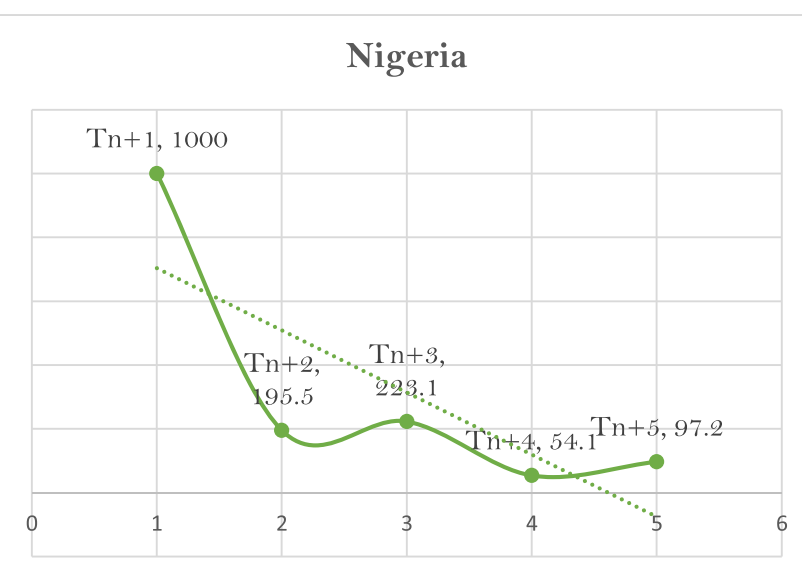

Fig. 4 Trends in COVID-19 cases in Nigeria

3). Conversely, the country experienced a sharp increase in the rate of reported cases, which stood at $99 \%$ in $(\mathrm{Tn}+4)$. A further increase in reported cases in Ghana, to $155.4 \%$, was then seen in $(\mathrm{Tn}+5)$. The growth rates for reported cases of COVID-19 for Niger Republic, as captured in Fig. 6, indicate a $0 \%$ growth rate in both $(\mathrm{Tn}+1)$ and $(\mathrm{Tn}+2)$. However, this was followed by an increase of $1340 \%$ in $(\mathrm{Tn}+3)$, a subsequent drop to $241 \%$ in $(\mathrm{Tn}+4)$, and a further decline to $32 \%$ in $(\mathrm{Tn}+5)$.

The cumulative results across countries of the West African region as captured in Fig. 7 indicate an increase of $303.1 \%$ in cases of COVID-19 in $(\mathrm{Tn}+1)$, followed by a decrease to $117.9 \%$ growth of reported cases recorded in $(\mathrm{Tn}+2)$. The growth rate in reported cases of infection then increased to $177.5 \%$ for the region in $(\mathrm{Tn}+3)$, and declined to $92.6 \%$ in $(\mathrm{Tn}+4)$. The growth rate in reported cases of COVID-19 in West Africa was $72.4 \%$ in $(\mathrm{Tn}+5)$. Globally, the pattern of growth in the incidence of COVID-19 as reported from ( $\mathrm{Tn}+$ $1)^{1}$ to $(\mathrm{Tn}+5)$ showed a downward slope. The results as captured in Fig. 8 indicate that the growth rate rose to $303.1 \%$ in $(\mathrm{Tn}+1)$ and dropped to $117.3 \%$ in $(\mathrm{Tn}+2)$. Similarly, a growth rate of $78.6 \%$ was reported in $(T n+3)$ and $49.6 \%$ in $(T n+4)$. The global rate of growth for the pandemic was $41.7 \%$ in ( $\mathrm{Tn}+$ 5).

The trend in deaths arising from COVID-19 infection was very similar for Burkina Faso, Nigeria, Ghana, Niger Republic, and West Africa. This is captured in Figs. 9, 12, 13,14 , and 15 , respectively. The trend in reported deaths for the Ivory Coast and Senegal followed the same pattern, as shown in Figs. 10 and 11, respectively. However, the global

\footnotetext{
${ }^{1}$ Note: $(\mathrm{Tn}+1)$ denotes the percentage growth in reported cases of COVID-19 from March 15 through March 22, 2020; $(\mathrm{Tn}+2)$ denote percentage growth in reported cases of COVID-19 from March 22 through March 29, 2020; $(\mathrm{Tn}+3)$ denotes percentage growth in reported cases of COVID-19 from March 29 through April 5, 2020; $(\mathrm{Tn}+4)$ denotes percentage growth in reported cases of COVID-19 from April 5, 2020, through April 12, 2020; and (Tn+5) denotes percentage growth in reported cases of COVID-19 from April 12, 2020, through April 19, 2020.
}

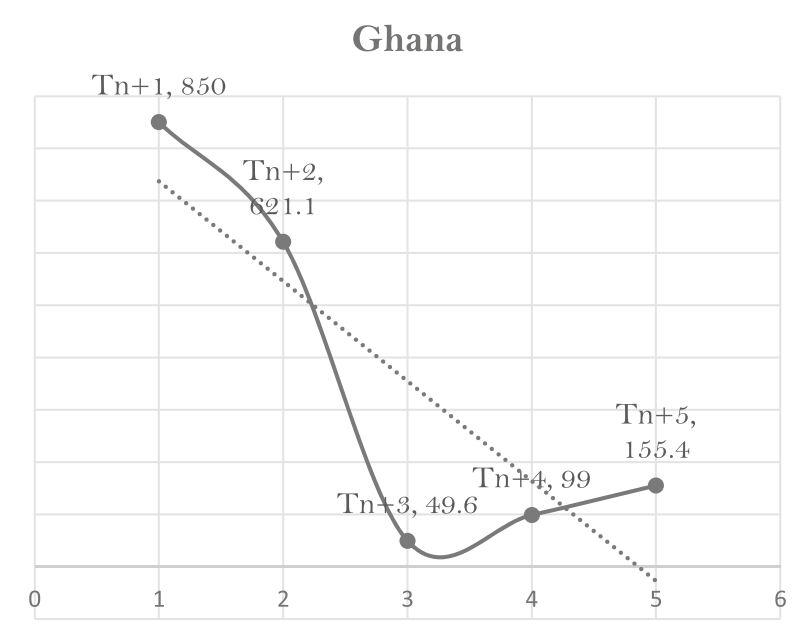

Fig. 5 Trends in COVID-19 cases in Ghana

trend in deaths from COVID-19 differed, and is shown in Fig. 16.

As indicated in Fig. 9, the growth rate for deaths arising from COVID-19 in Burkina Faso increased from $0 \%$ in $(\mathrm{Td}+$ 2) to $3400 \%$ in $(\mathrm{Td}+3)$. No deaths $(0 \%)$ due to COVID-19 infection were reported in $(\mathrm{Td}+1)$ or $(\mathrm{Td}+2)$ in Burkina Faso. The results also show that the growth in the country's death rate dropped to $36.7 \%$ in $(\mathrm{Td}+4)$, but then increased to $89.5 \%$ in $(\mathrm{Td}+5)$. The results for Ivory Coast as captured in Fig. 10 show that the death rate increased from $0 \%$ in $(\mathrm{Td}+3)$ to $4100 \%$ in $(\mathrm{Td}+4)$; no deaths were reported in $(\mathrm{Td}+1),(\mathrm{Td}+$ $2)$, or $(\mathrm{Td}+3)$. An increase in deaths of $5125 \%$ was reported in $(\mathrm{Td}+5)$ as a result of the spread of the pandemic in the Ivory Coast.

Figure 11 shows that for Senegal, there was a 0\% growth rate in deaths arising from COVID-19 infection in $(\mathrm{Td}+1)$, $(\mathrm{Td}+2)$, and $(\mathrm{Td}+3)$. Recorded deaths due to COVID-19 complications for the country increased from $0 \%$ in $(\mathrm{Td}+4)$ to $50 \%$ in $(\mathrm{Td}+5)$. The results for Nigeria as captured in Fig. 12

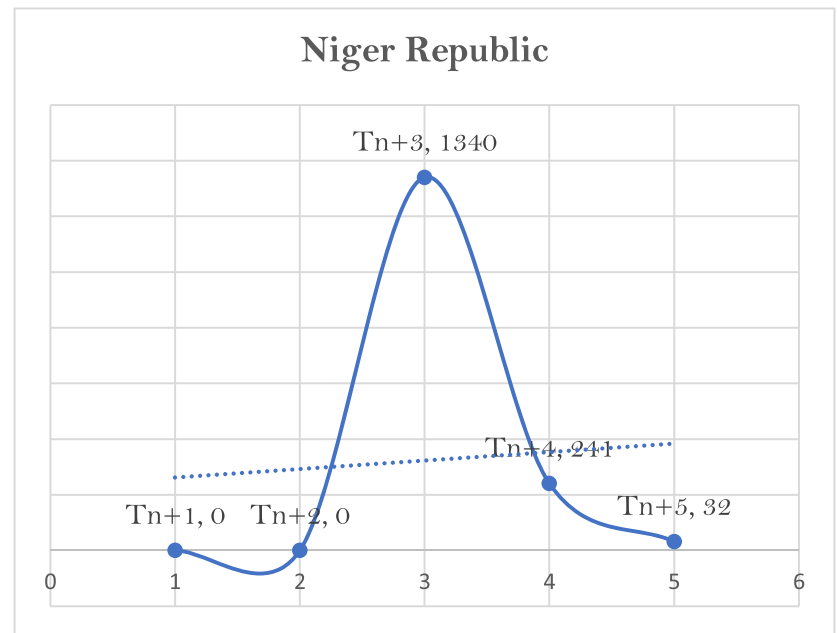

Fig. 6 Trends in COVID-19 cases in Niger Republic 


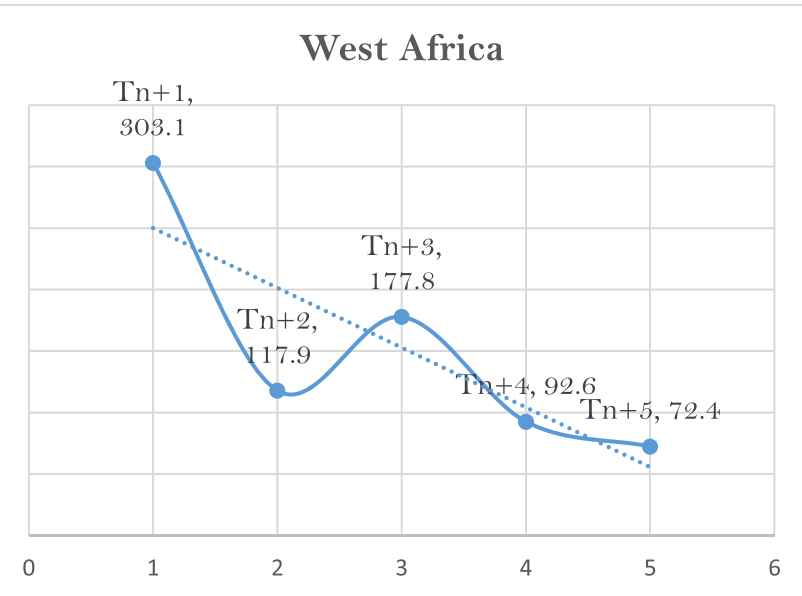

Fig. 7 Trends in COVID-19 cases in West Africa

indicate that the death rate increased from $0 \%$ in $(\mathrm{Td}+2)$ to $3300 \%$ in $(\mathrm{Td}+3)$. The earlier pattern showed $0 \%$ growth in deaths as a result of the infection in $(\mathrm{Td}+1)$ and $(\mathrm{Td}+2)$. However, the death rate was estimated at $110 \%$ in $(\mathrm{Td}+5)$.

Results for Ghana as captured in Fig. 13 show that a $0 \%$ rate in deaths related to COVID-19 infection was recorded in the country in $(\mathrm{Td}+1)$ and $(\mathrm{Td}+2)$. A $25 \%$ increase in the death rate was reported in $(\mathrm{Td}+3$.). The rate increased to $60 \%$ in $(\mathrm{Td}+4)$ and subsequently dropped to $12.5 \%$ in $(\mathrm{Td}+5$.$) . As$ captured in Fig. 14, the rate of deaths arising from the COVID-19 pandemic in the Republic of Niger was $0 \%$ in $(\mathrm{Td}+1)$ and $(\mathrm{Td}+2)$. The rate increased to $3700 \%$ in $(\mathrm{Td}+3)$ but subsequently dropped to $37.5 \%$ in $(\mathrm{Td}+4)$. The rate rose to $81.8 \%$ for the country in $(\mathrm{Td}+5)$.

The pattern of deaths for West Africa showed an initial upward movement from $0 \%$ in $(\mathrm{Td}+1)$ to $566.7 \%$ in $(\mathrm{Td}+2)$, as captured in Fig. 15. Deaths arising from the COVID-19 pandemic then dropped to $135 \%$ for the sub-region in $(\mathrm{Td}+$ 3 ) and further declined to $57.4 \%$ in $(\mathrm{Td}+4)$, but subsequently increased to $135.1 \%$ in $(\mathrm{Td}+5)$. The global rate of deaths arising from the pandemic dropped from $1405.1 \%$ in $(\mathrm{Td}+1)^{2}$ to $133.8 \%$ in $(\mathrm{Td}+2)$, as captured in Fig. 16. This was followed by a further decline in reported COVID-19-related deaths to $110 \%$ and $68.8 \%$ in $(\mathrm{Td}+3)$ and $(\mathrm{Td}+4)$, respectively. The rate then dropped from $68.8 \%$ in $(\mathrm{Td}+4)$ to $55.7 \%$ in $(\mathrm{Td}+5)$.

Table 1 presents the results of the analysis of the association between the incidence of COVID-19 infection and deaths arising from its complications across the studied countries and regions. The results show that complications of COVID-19 infection accounted for 92.3\%, 97.8\%, 90.3\%, 65\%, 90.4\%, and $93.6 \%$ of patient deaths in Burkina Faso, Nigeria,

\footnotetext{
${ }^{2}$ Note: $(\mathrm{Td}+1)$ denotes percentage increase in deaths due to the COVID-19 pandemic from March 15 through March 22, 2020; $(\mathrm{Td}+2)$ denotes the percentage from March 22 through March 29, 2020; $(\mathrm{Td}+3)$ denotes the percentage from March 29 through April 5, 2020; $(\mathrm{Td}+4)$ denotes the percentage from April 5 through April 12, 2020; and ( $\mathrm{Td}+5)$ denotes the percentage from April 12 through April 19, 2020.
}



Fig. 8 Trends in COVID-19 cases in the world

Senegal, Ghana, Niger Republic, and Ivory Coast, respectively. Also, the pandemic accounted for $97 \%$ and $98.9 \%$ of complications leading to the death of patients in the sub-region of West Africa and the world at large. The results further reveal that the increase in deaths arising from COVID-19 complications is positively and strongly associated. Evidence from the study as presented in Table 1 showed a strong positive linear association between COVID-19 incidence and deaths arising from its complications among patients in Burkina Faso $(r=$ $0.968)$, Nigeria $(r=0.978)$, Senegal $(r=0.961)$, Ghana $(r=$ $0.849)$, Niger $(r=0.849)$, and Ivory Coast $(\mathrm{r}=0.974)$. Likewise, a strong positive linear association was found between the spread of COVID-19 and an increase in deaths related to its complications among patients in the West African sub-region $(r=0.988)$ and the world at large $(r=$ 0.996).

Correspondingly, the results consistently showed a significant association between the spread of COVID-19 and deaths arising from its complications among patients in the studied countries and regions, including Burkina Faso $(\beta=0.059 ; t=$ $7.74 ; p<0.01)$, Nigeria $(B=0.034 ; t=14.95 ; p<0.001)$, Senegal $(\beta=0.009 ; t=6.90 ; p<0.01)$, Ghana $(\beta=0.008 ; t=$

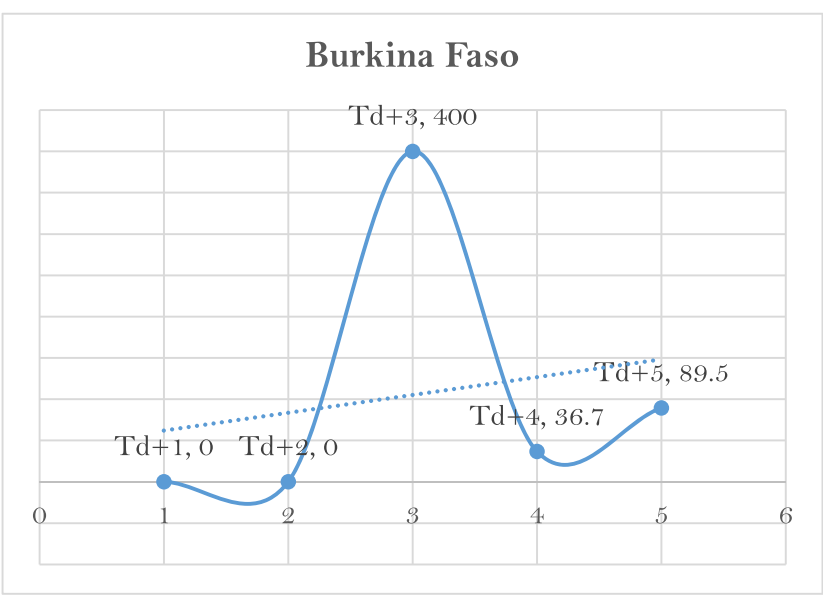

Fig. 9 Trends in COVID-19-related deaths in Burkina Faso 


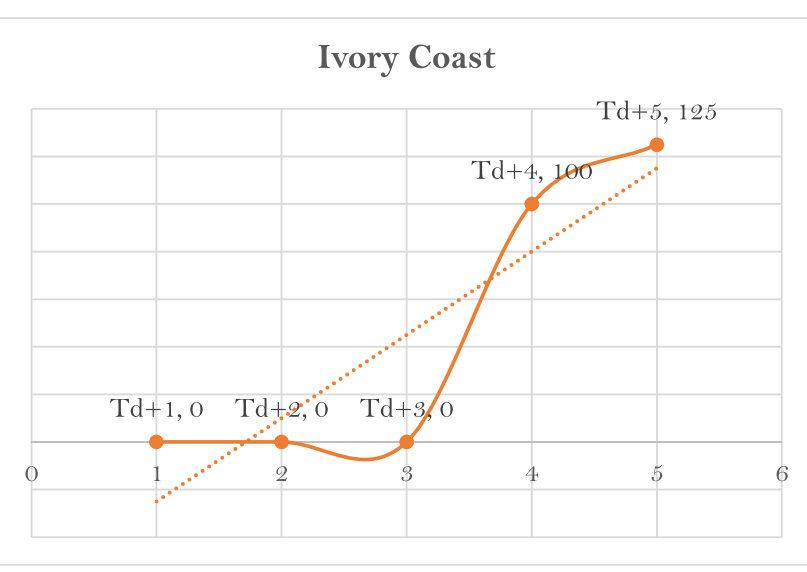

Fig. 10 Trends in COVID-19-related deaths in Ivory Coast

$3.21 ; p<0.05)$, Niger Republic $(\beta=0.027 ; t=6.94 ; p<0.01)$, and Ivory Coast $(\beta=0.010 ; t=8.59 ; p<0.01)$. A similar association was demonstrated in West Africa $(\beta=0.032 ; t=$ $12.70 ; p<0.001)$ and globally $(\beta=0.070 ; t=21.01$; $p<0.001)$.

\section{Discussion}

This study comprehensively investigated the trends in the spread of the COVID-19 pandemic in Burkina Faso, Ghana, Nigeria, Ivory Coast, Senegal, and Republic of Niger. The study also describes the spread of COVID-19 in the West African sub-region as a whole and the world at large. Trends in deaths arising from COVID-19 infection among patients are also described in the study. Moreover, the study establishes a link between the spread of the COVID-19 pandemic and the increase in deaths arising from the spread of the infection. Our study shows that the spread of COVID-19 in the subregion of West Africa increased gradually. For instance, we observed that the rate of spread varied across the six countries encompassing West Africa; nevertheless, all the countries

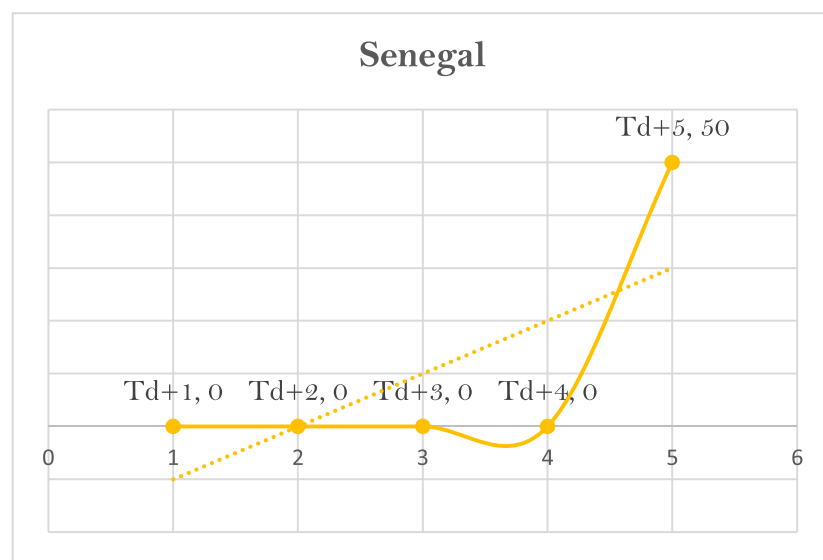

Fig. 11 Trends in COVID-19-related deaths in Senegal

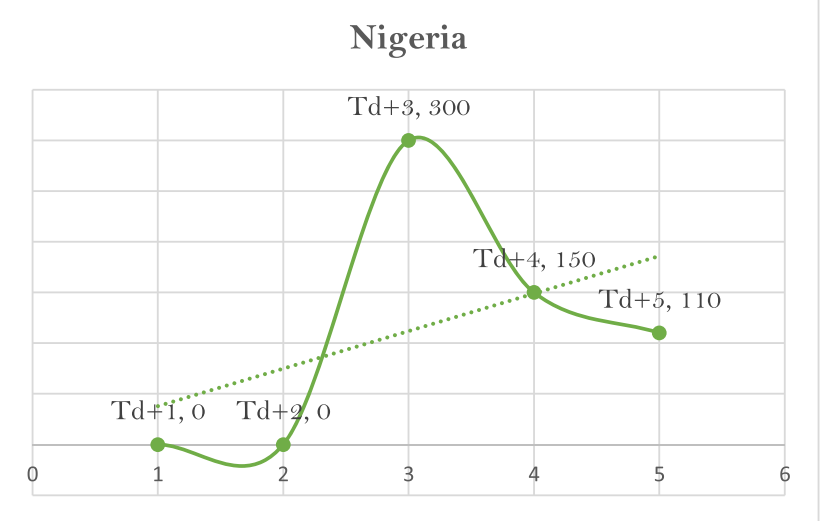

Fig. 12 Trends in COVID-19-related deaths in Nigeria

recorded growth in reported cases within the time frame covered in the study.

In Burkina Faso, the spread of the virus peaked in $\mathrm{Tn}+1$ $(2300 \%)$, while the lowest growth rate of $30 \%$ was reported in $\mathrm{Tn}+2$. The growth trend in Nigeria followed the same pattern. However, while the highest number of reported COVID-19 cases was recorded in $\mathrm{Tn}+1(1000 \%)$, the lowest growth in the spread of the infection was reported in $\mathrm{Tn}+4(54.1 \%)$. Our findings for the situation in Senegal showed that the highest spread in the country was reported in $\mathrm{T} n+1(166.7 \%)$, while the lowest reported case spread was recorded in $\mathrm{Tn}+4(26 \%)$. We observed that the growth in the spread of COVID-19 in the Ivory Coast peaked in $\mathrm{Tn}+2(1445.6 \%)$ and was lowest in $\mathrm{Tn}+5(53.2 \%)$. Our findings further showed that in Ghana, the highest growth in COVID-19 incidence was recorded in $\mathrm{Tn}+1$ $(1850 \%)$, while the lowest growth rate was reported in $\mathrm{Tn}+3$ (49.6\%). In contrast to Ghana, in the Republic of Niger we observed that the lowest COVID-19 pandemic growth rate was recorded in $\mathrm{Tn}+5$ (32\%), while the growth rate peaked in $\mathrm{Tn}+3(1340 \%)$. Collectively, the growth rate in West Africa was highest in $\mathrm{Tn}+1(303.1 \%)$, while the lowest growth in the sub-region was observed in $\mathrm{Tn}+5(72.4 \%)$. We found that the

\section{Ghana}

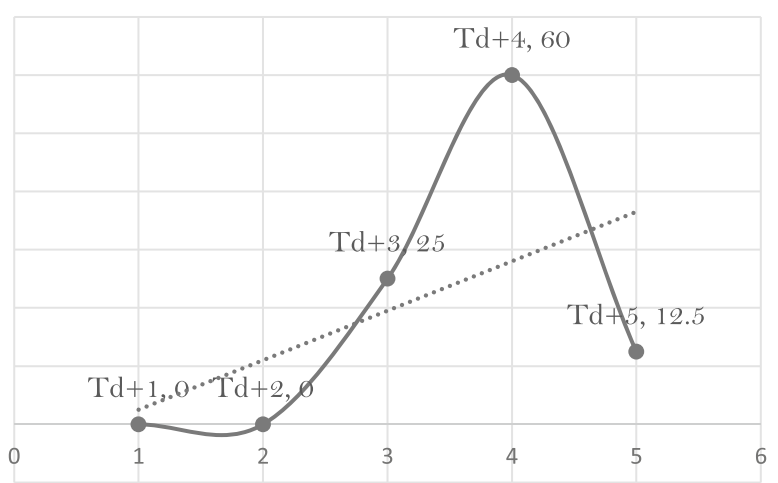

Fig. 13 Trends in COVID-19-related deaths in Ghana 


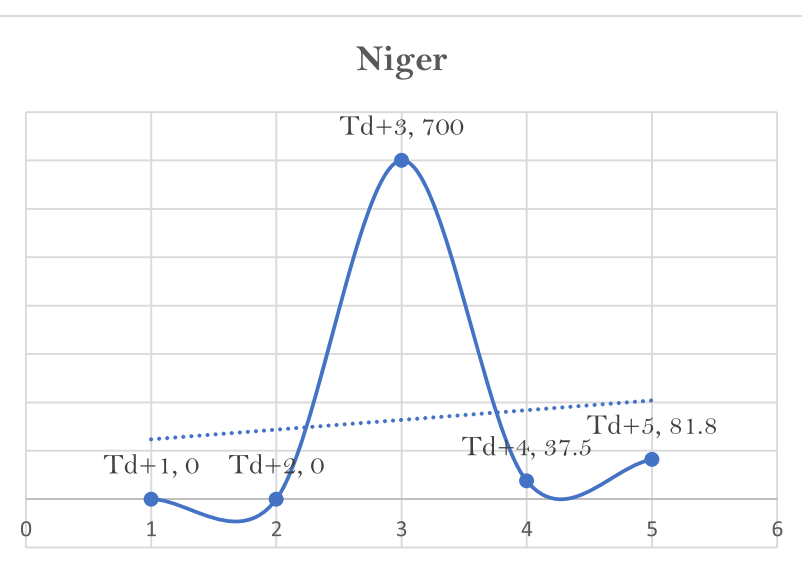

Fig. 14 Trends in COVID-19-related deaths in Niger

trend in growth globally peaked in $\mathrm{Tn}+1(303.1 \%)$, while the lowest growth was recorded in $\mathrm{Tn}+5(41.7 \%)$.

Evidence from our study showed a continuous decline in the growth rate of the spread of the COVID-19 pandemic globally, while the reverse was observed in the sub-region of West Africa. Thus, our findings showed a fluctuating trend in the spread of the infection in West Africa. Specifically, the trends of growth rates in the spread of COVID-19 for Ghana, Nigeria, Burkina Faso, Senegal, Niger Republic, and Ivory Coast vary over the period of the study. Hence, it must be deduced from our findings that the lower cases of COVID19 infection reported in West Africa as at April 19, 2020, could not be attributed to the existence of a better, functional, and improved healthcare service delivery compared to that obtainable in the developed regions of the world. Based on our findings, if the healthcare service had been the major factor for a lower number of deaths in the region compared to the alarming global figure, the growth rate should also be declining rather than the rise witnessed in Ghana, Nigeria, Senegal, Ivory Coast, Niger Republic, Burkina Faso, and the subregion of West Africa as a whole.



Fig. 15 Trends in COVID-19-related deaths in West Africa

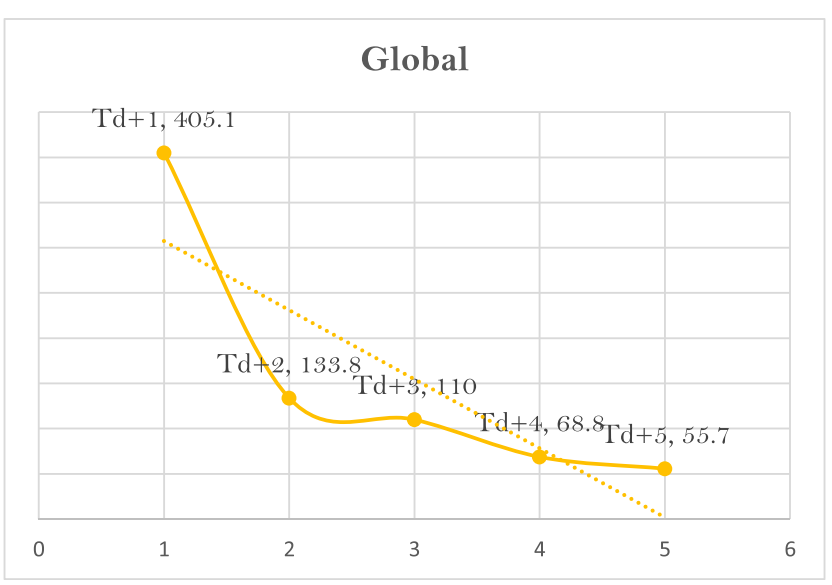

Fig. 16 Trends in COVID-19-related deaths in the world

In fact, the lower number of reported cases of COVID-19 infection in West Africa could also be attributable to the lower number of tests that have been carried out so far, which agrees with the prediction by Melinda Gates as reported by Sahara Reporters on April 14, 2020. Our assertion is supported by the fact that as of April 19, 2020, a total of 35, 131, 195, and 510 COVID-19 tests per million population had been carried out in Niger, Nigeria, Gambia, and Togo, respectively (Worldometer 2020). Moreover, evidence from contemporary studies indicates that the state of healthcare service delivery in the sub-Saharan African region is characterised by poor funding, shortage of modern sophisticated healthcare equipment, poor or absent healthcare insurance schemes, shortage of well-trained healthcare personnel, and poor implementation of healthcare policies (Azevedo 2017; Doctor et al. 2018; Kiri and Ojule 2020; Lawumi 2020; Oleribe et al. 2019; WHO 2020a).

We observed from our study that globally, the trends in deaths arising from the COVID-19 pandemic has been steadily declining. Growth in deaths among COVID-19 patients peaked in $\mathrm{Td}+1(1405.1 \%)$, while the lowest growth rate in deaths was reported in $\mathrm{Td}+5(55.7 \%)$. Unlike the trend in deaths arising from the COVID-19 pandemic in the world at large, we observed a rise and fall in COVID-19-related deaths among patients in Ghana, Nigeria, Senegal, Ivory Coast, Burkina Faso, and Niger Republic, and in the region of West Africa. For instance, we observed that the death rate growth in West Africa peaked in $\mathrm{Td}+2(566.7 \%)$ and was lowest in $\mathrm{Td}+4(57.4 \%)$. Specifically, we observed that the growth in deaths arising from COVID-19 complications among patients in Ghana peaked in $\mathrm{Td}+1(60 \%)$, while the lowest growth in deaths was reported in $\mathrm{Td}+3(25 \%)$. Also, we discovered that growth rates in deaths arising from COVID-19 infection among patients in Niger peaked in $\mathrm{Td}+$ $3(700 \%)$, while the lowest growth rate in deaths was witnessed in $\mathrm{Td}+4(37.5 \%)$.

Our findings showed that deaths arising from COVID-19 infection peaked among patients in Burkina Faso and Ivory 
Table 1 Results of correlation coefficient and regression analysis showing the cause-effect association and correction coefficient between COVID-19 pandemic and deaths in West Africa

\begin{tabular}{lllllllll}
\hline Variables & Burkina Faso & Nigeria & Senegal & Ghana & Niger & Ivory Coast & West Africa & Global \\
\hline $\mathrm{T}_{\mathrm{n} 1 \_\mathrm{n} 6}$ & $\mathrm{~T}_{\mathrm{d} 1 \_\mathrm{d} 6}$ & $\mathrm{~T}_{\mathrm{d} 1 \_\mathrm{d} 6}$ & $\mathrm{~T}_{\mathrm{d} 1 \_\mathrm{d} 6}$ & $\mathrm{~T}_{\mathrm{d} 1 \_\mathrm{d} 6}$ & $\mathrm{~T}_{\mathrm{d} 1 \_\mathrm{d} 6}$ & $\mathrm{~T}_{\mathrm{d} 1 \_\mathrm{d} 6}$ & $\mathrm{~T}_{\mathrm{d} 1 \_\mathrm{d} 6}$ \\
$\beta$ & 0.059 & 0.034 & 0.009 & 0.008 & 0.027 & 0.010 & 0.032 & 0.070 \\
Standard error & 0.008 & 0.002 & 0.001 & 0.003 & 0.004 & 0.001 & 0.003 & 0.003 \\
$t_{c}$ & 7.74 & 14.95 & 6.90 & 3.21 & 6.94 & 8.59 & 12.70 & 21.01 \\
$p$ value & $0.001^{* *}$ & $0.000^{* * *}$ & $0.002^{* *}$ & $0.033^{*}$ & $0.002^{* *}$ & $0.001 * *$ & $0.000^{* * *}$ \\
$r$ & $0.968^{*}$ & $0.991^{*}$ & $0.961^{*}$ & $0.849 *$ & $0.961^{*}$ & $0.974 *$ & $0.000^{* * *}$ \\
$R_{0}$ & 0.923 & 0.978 & 0.903 & 0.650 & 0.904 & 0.936 & $0.988^{*}$ & $0.996^{*}$ \\
\hline
\end{tabular}

Note: *Significant at $p<0.05, * *$ significant at $p<0.01, * * *$ significant at $p<0.001 ; R_{0}=$ adjusted $r^{2} ; r=$ correlation coefficients; $\beta=$ regression coef-

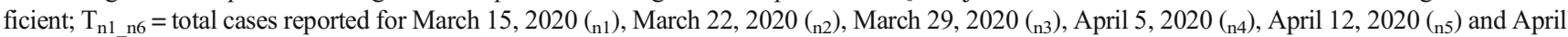
19, $2020\left({ }_{\mathrm{n} 6}\right) ; \mathrm{T}_{\mathrm{d} 1 \mathrm{~d} 6}=$ total deaths reported for March 15, 2020 (d1), March 22, 2020 (d2), March 29, 2020 (d3), April 5, 2020 (d4), April 12, (d5) and April 19,2020 respectively

Coast in $\mathrm{Td}+3(3400 \%)$ and $\mathrm{Td}+5(5125 \%)$, while the lowest growth in the rate of deaths arising from the pandemic was reported in Td+4 (36.7\%) and Td+4 (4100\%), respectively. In Nigeria, the death rate peaked in $\mathrm{Td}+3(300 \%)$, and the lowest rate was recorded in $\mathrm{Td}+5(110 \%)$. Likewise, the death rate in Senegal peaked in $\mathrm{Td}+5$ (50\%). Evidence from our study showed that the growth rate of deaths arising from COVID19 complications has been steadily declining globally. However, the reverse was the case for death growth rates for Ghana, Nigeria, Ivory Coast, Burkina Faso, Senegal, Niger Republic, and West Africa.

Our findings, therefore, suggest that if the relatively lower incidence of growth in the reported cases of COVID-19 in West Africa is as a result of the availability of an improved or functional healthcare delivery system, the number of deaths across covered countries and of the region as a whole should have declined gradually rather than the present gradually increasing rate of deaths that are being recorded. Based on our findings, it is possible that cases of COVID-19 are being significantly underreported or not reported at all. This could be due largely to the poor healthcare delivery system in many of the regional countries.

In fact, our findings consistently showed the existence of a significant association between increased rates of reported cases of COVID-19 and an increase in the rate of deaths associated with complications arising from the disease. Our findings are supported by Zhao et al. (2020), who posited that cases of COVID-19 were being underreported in China. Also, our observation that it is possible that COVID-19 cases in West Africa have been significantly underreported as a result of the poor state of healthcare service delivery in the region was corroborated by $\mathrm{Wu}$ et al. (2020) and Chen et al. (2020), who argued that the absence of substantial public health interventions could bring about an unanticipated spread of the virus outside China.

\section{Conclusion}

The threat posed by the COVID-19 pandemic seems to be low in many countries in West Africa and sub-Saharan Africa in comparison to what is being witnessed in other regions of the world. This study demonstrated in detail that the present situation in the region may not be as a result of the availability and accessibility of functional or improved healthcare facilities for nationals in the region. Therefore, the reason for the current slower trends in terms of growth rates of reported cases or deaths could be a result of lack of test kits, underreported cases, and the like. In fact, it is unlikely that many of the governments in this region could provide their nationals with adequate palliatives that are needed at this trying time. Hence, the study concludes that time will eventually tell whether the region is prepared for the challenges that may arise from the pandemic should it spiral out of control. Certainly, there is a need for the nations of the region to be fully prepared to execute measures to contain the spread of the COVID-19 pandemic.

\section{Policy implications}

Based on the observed pattern of increased incidence of COVID-19 and the increase in the proportion of recorded deaths over the time covered in this study, the authors recommend that the lockdown measures undertaken by governments in the region be fully implemented. Also, in order to achieve total compliance with lockdown, regional governments should provide their nationals with palliatives in the form of food, drugs, preventive supplies (face masks, hand sanitizers), potable water, and other basic necessities of life. Healthcare service delivery should be made available and at no cost to the public. In fact, the governments, and in collaboration with richer and developed countries, should work 
towards covering a wider range of the population. Hence, there is a need to carry out more testing. There is a need to set up more quarantine centers, especially in high-risk areas.

Equally, additional intensive-care units should be established along with the required healthcare equipment such as ventilators, immunity-boosting drugs and the like. Moreover, medical professional support should be sought from countries with a high COVID-19 recovery rate. Hence, there is an urgent need to seek medical support from Germany, New Zealand, Australia, South Korea, Austria, Iceland, Iran, Thailand, and China. Finally, huge spending on healthcare service delivery and research, particularly in health science and technology, is critical, along with the establishment of functional and well-funded social and healthcare insurance schemes.

Acknowledgements The authors express their gratitude to the World Health Organization (WHO) for making the datasets available for use (Granted Permission Number: 330569). Also, the Worldometer is acknowledged for usage of their data.

Authors' contributions TOB developed the concept, analysed data and interpreted the results. OBO, AOO and DOA reviewed the literature. All the authors were involved in the discussion of findings. All authors read and approved the final manuscript.

\section{Compliance with ethical standards}

Conflict of interest The authors declare no competing interests.

Data authorisation Please contact the corresponding author for the generated data request.

Ethical consideration The ethical issue of the study has already been met, since the datasets that were utilized in this study were extracted from the 2020 COVID-19 World Health Organization Databank.

\section{References}

Andrea R, Giuseppe R (2020) COVID-19 and Italy: what next? Lancet 395:1225-1228. https://doi.org/10.1016/S0140-6736(20)30627-9

Azevedo MJ (2017). The State of Health System(s) in Africa: challenges and opportunities In: Historical Perspectives on the State of Health and Health Systems in Africa (ed). Vol. II. Cham: Palgrave Macmillan, pp 1-73

Chen T, Rui J, Wang Q, Zhao Z, Cui J, Yin L (2020) A mathematical model for simulating the phase-based transmissibility of a novel coronavirus. Infect Dis Poverty 9(24):1-8. https://doi.org/10.1186/ s40249-020-00640-3

Doctor HV, Nkhana-Salimu S, Abdulsalam A (2018) Health facility delivery in sub-Saharan Africa: successes, challenges, and implications for the 2030 development agenda. BMC public health 18(1) 765: $1-12$

Gautier L, Ridde V (2017) Health financing Policies in sub-Saharan Africa: Government Ownership or Donors' influence? A Scoping Review of Policymaking Processes. Global Health Res Policy 2017 2:23

Kiri VA, Ojule AC (2020) Electronic medical record systems: a pathway to sustainable public health insurance schemes in sub-Saharan
Africa. Nigerian Postgraduate Med J 27(1):1-7. https://doi.org/10. 4103/npmj.npmj

Lawumi A (2020) Health Insurance: The Theoretical Basis http://www. nursingworldnigeria.com/2015/01/health-insurancethe-theoreticalbasis-by-dr-lawumi-adekola. Accessed 14 May 2020

Li M, Zhang Z, Jiang S, Liu Q, Chen C (2020) Predicting the epidemic trend of COVID-19 in China and across the world using the machine learning approach. J medRxiv. https://doi.org/10.1101/2020.03.18. 20028117

McLeroy KR, Bibeau D, Steckler A, Glanz K (1988) An ecological perspective on health promotion programs. Health Education Quarterly 15:351-377

Oleribe OO, Momoh J, Uzochukwu BS, Mbofana F, Adebiyi A, Barbera T et al (2019) Identifying Key Challenges Facing Healthcare Systems in Africa and Potential Solutions. Int J Gen Med 12:395-403

Olugbenga EO (2017) Workable Social Health Insurance Systems in subSaharan Africa: Insights from Four Countries. J African Develop 42: $147-175$

Saharan Report (2020) Melinda Gates Predicts High Coronavirus Deaths in Africa by Sahara Reporters, New York Apr 14, 2020. http:// saharareporters.com/2020/04/14/melinda-gates-predicts-highcoronavirus-deaths-africa. Accessed April 23, 2020

World Health Organization (2019) World Population prospect - Data booklet https://www.google.com/search?client=firefox-b-d\&q= World+Population+Prospects+2019+\%E2\%80\%A2+Data+ Booklet

World Health Organization. (2020a) Infection prevention and control during health care when COVID-19 is suspected: interim guidance, 19 April 2020 (No. WHO/2019-nCoV/IPC/2020.3). World Health Organization. https://www.who.int/publications-detail/infectionprevention-and-control-during-health-care-for-probable-orconfirmed-cases-of-middle-east-respiratory-syndrome-coronavirus(mers-cov)-infection)Accessed April 15, 2020

World Health Organization (2020b). Infection prevention and control during healthcare when COVID-19 is suspected. Interim Guidance WHO, (2020). WHO reference number: WHO/2019-nCoV/IPC/ 2020.3? https://www.preventionweb.net/publications/view/71051. Accessed on April 15, 2020

World Health Organization (2020c) COVID-19 situations data. Accessed on April, 2020 https://www.who.int/emergencies/diseases/novelcoronavirus-2019/situatio

World Health Organization (2020d) Laboratory testing for coronavirus disease 2019 (COVID-19) in suspected human cases: interim guidance, 2 March 2020 (no. WHO/COVID-19/laboratory/2020.4). World Health Organization. https://apps.who.int/iris/handle/10665/331329

Worldometer (2020) COVID-19 Data Report. https://www. worldometers.info/coronavirus/

Wu JT, Leung K, Leung GM (2020) Nowcasting and forecasting the potential domestic and international spread of the 2019-nCoV outbreak originating in Wuhan, China: a modelling study. Lancet 395(10225):689-697. https://doi.org/10.1016/S0140-6736(20) 30260-9

Zhao S, Musa SS, Lin Q, Ran J, Yang G, Wang W et al (2020) Estimating the Unreported Number of Novel Coronavirus (2019-nCoV) Cases in China in the First Half of January 2020 : A Data-Driven Modelling Analysis of the Early Outbreak, J Clin Med, 9(2): https://doi.org/10.3390/jcm9020388

Zhou P, Yang X, Wang X, Hu B, Zhang L, Zhang W et al (2020) A pneumonia outbreak associated with a new coronavirus of probable bat origin. Springer Nat 579(7798):270-273. https://doi.org/10. 1038/s41586-020-2012-7

Publisher's note Springer Nature remains neutral with regard to jurisdictional claims in published maps and institutional affiliations. 